\title{
Higher frequency of hepatic steatosis at CT among COVID-19-positive patients
}

\author{
Augusto Kreling Medeiros ${ }^{1}$. Cinthia Callegari Barbisan ${ }^{1} \cdot$ Italo Ribeiro Cruz $^{1} \cdot$ Eduardo Medeiros de Araújo $^{1}$. \\ Bruna Brandão Libânio ${ }^{1} \cdot$ Kamila Seidel Albuquerque $^{1} \cdot$ Ulysses S. Torres $^{2}$ (i)
}

Received: 10 June 2020 / Revised: 1 July 2020 / Accepted: 9 July 2020 / Published online: 18 July 2020

(c) Springer Science+Business Media, LLC, part of Springer Nature 2020

\begin{abstract}
Purpose Recent studies have demonstrated that obesity is significantly associated with increased disease severity, hospitalizations and mortality in COVID-19, with a potential role in the pathogenesis and prevalence in the new pandemic. The association with hepatic steatosis, however, a condition closely related to obesity within the spectrum of systemic metabolic dysfunctions, remains to be elucidated. We aimed to evaluate the frequency of hepatic steatosis as incidentally detected in chest CT examinations of COVID-19 positive patients in comparison to non-infected controls.

Methods A retrospective study was performed with 316 patients (204 RT-PCR positive; 112 RT-PCR negative and chest CT negative). Steatosis was measured with placement of a single ROI in the right lobe of the liver (segments VI-VII) and defined as a liver attenuation value $\leq 40 \mathrm{HU}$.

Results The frequency of hepatic steatosis was higher in the RT-PCR positive group in comparison to controls (31.9\% vs. $7.1 \%, p<0.001)$. Logistic linear regression analysis showed a 4.7 times odds of steatosis in the COVID-19 positive group as compared to controls after adjusting for age and sex (OR 4.698; 95\% IC 2.12-10.41, $p<0.001$ ).

Conclusion A significantly higher prevalence of steatosis was found among COVID-19 positive individuals. These findings are in accordance with other recent studies linking obesity and COVID-19 infection, as there is an intricate relationship between liver steatosis, metabolic syndrome and obesity. Further studies are required to confirm if such association remains after accounting for multiple variables, as well as possible relationships with disease severity and worst clinical outcomes.
\end{abstract}

Keywords Hepatic steatosis $\cdot$ COVID-19 $\cdot$ CT $\cdot$ Liver

\section{Introduction}

On March 11, 2020, the World Health Organization declared Coronavirus disease 2019 (COVID-19) a pandemic, with more than 10.5 million cases reported in more than 200 countries by July 1, 2020. The features most commonly associated with acute respiratory syndrome coronavirus-2 (SARS-CoV2) are male sex, older age, cardiovascular disease and diabetes [1]. Several recent studies, however, have been demonstrating that obese persons are more likely to progress to severe pneumonia due to SARS-CoV2 infection

Ulysses S. Torres

ulysses.torres@grupofleury.com.br

1 Hospital Beneficência Portuguesa, Sao Paulo, Brazil

2 Grupo Fleury, Rua Cincinato Braga, 282, Bela Vista, São Paulo, SP 01333-010, Brazil when compared to normal weight individuals, even after adjusting for co-morbidities and other risk factors; obesity also has been associated to a greater risk of needing hospitalizations, mechanical ventilation due to acute respiratory failure, extensive coagulopathy, and, finally, death [2].

Obesity is at epidemic levels in the United States and in most of the Westernized World: nearly three-fourths of adults over the age of 20 years in the US meet criteria for being overweight or obese, and $42 \%$ are obese [body mass index $(\mathrm{BMI}) \geq 30 \mathrm{~kg} / \mathrm{m}^{2}$ ] [3]. The relationship between obesity and respiratory disease is largely known: weight excess is positively associated with asthma, obstructive sleep apnea syndrome, and obesity-hypoventilation syndrome [4]. Since obesity has been shown to increase vulnerability to infections and as obesity-induced adipose tissue chronic inflammation resulting from metabolic dysfunction has deleterious effects on the immune system, the disease is thought to play a crucial role in the pathogenesis and prevalence of 
COVID-19 infection, possibly as an independent risk factor [3, 5-7].

While this association has been assessed for obesity, the same does not apply for hepatic steatosis, currently one of the most important conditions within the wide range of systemic metabolic dysfunctions triggered by obesity. Indeed, with the continued rise of obesity in the Western countries, the prevalence of liver steatosis has followed a similar trend, as it is closely associated with visceral obesity and excessive body mass index [8].

Although liver biopsy and histologic analysis is considered the diagnostic reference standard for the assessment of hepatic steatosis [8], a non-invasive diagnosis may be alternatively reached by means of cross-sectional imaging [9]. At unenhanced CT, the normal liver has slightly greater attenuation than the spleen and blood, and intrahepatic vessels are visible as relatively hypoattenuated structures. Hepatic steatosis can be diagnosed if the attenuation of the liver is at least $10 \mathrm{HU}$ less than that of the spleen or if the attenuation of the liver is less than $40 \mathrm{HU}$ [9].

With the crescent role of chest $\mathrm{CT}$ in the management of COVID-19 (as imaging manifestations are extremely helpful in the early detection and diagnosis, in some cases even preceding a false-negative RT-PCR test result) [10], it is quite feasible to assess the liver parenchyma in the slices obtained at the thoracoabdominal junction in these patients, and radiologists are at a key position, therefore, to evaluate the organ for the presence of steatosis according to noninvasive diagnostic criteria.

By knowing the growing association between obesity and COVID-19 pointed by recent studies, we performed the present study to verify if such association could also be found for liver steatosis as detected in chest CT imaging of COVID-19 confirmed patients in comparison to confirmed negative controls.

\section{Patients and Methods}

This is a retrospective study conducted at the Radiology Department of our Institution from March 30, 2020 to April 17, 2020. The study was approved by the Institution Ethics and Research Committee (Registration number 32622720.9.0000.5483). Informed consent was waived given the retrospective nature and characteristics of the study.

We included 411 consecutive patients with flu syndrome and clinically suspected of having COVID-19 infection who underwent both chest CT and RT-PCR for a case-control study. All CT examinations were performed in the same scanner, a Somatom Perspective 64-slice (Siemens Healthcare, Forchheim, Germany). The technical parameters used in the CT exams were: collimation $64 \times 0.6 \mathrm{~mm}$, gantry rotation time $0.48 \mathrm{~s}$, recon slice width $8 \mathrm{~mm}$, pitch $1.25,110$
$\mathrm{KVp}$, mAs dependent on automatic modulation of the dose radiation (CARE Dose 4D by Siemens Healthcare).

From this number of patients, we excluded 11 patients who could have a bias in the liver assessment: three patients due to important artifacts in CT (the arms were lowered along the body), three patients who did not have a noncontrast-enhanced phase, two patients with polycystic liver disease, one patient with partial hepatectomy, one with right lobe hepatic atrophy due to portal venous thrombosis and one patient with liver transplantation.

The case group was composed by patients with a positive RT-PCR test for COVID-19 and the control group by patients with a negative RT-PCR test. However, as there is evidence that an early false-negative RT-PCR may occur in infected patients already showing CT manifestations of the disease (i.e., a positive CT) [10], to assure the criteria of negativity of the control group, we additionally checked all chest CT images of these individuals; those displaying CT findings within typical, atypical or indeterminate categories (according to the proposed reporting language for CT findings related to COVID-19 by the Radiological Society of North America (RSNA) [11]) were excluded, remaining only those who were considered 'negative". Further 84 individuals were excluded from the control group according to these criteria.

Finally, the case group (RT-PCR positive) was composed by 204 patients and the control-group (RT-PCR negative and chest CT negative pattern) by 112 patients, totaling 316 patients.

\section{CT evaluation}

Although there are several criteria for the assessment of hepatic steatosis by computed tomography (CT), most consider that an attenuation value of $\leq 40$ Hounsfield units (HU), measured in the region of interest (ROI), commonly in the right hepatic lobe in the non-enhanced phase, demonstrates a correlation with a pathological fat content of $\geq 30 \%$, suggesting at least moderate hepatic steatosis [12-14]. Another important evaluation criterion is the comparison with the ROI in the splenic parenchyma: when the liver attenuation is at least $10 \mathrm{HU}$ less than that of the spleen, steatosis is considered. Non-enhanced CT has a sensitivity to steatosis ranging from 43 to $95 \%$ and a specificity from 90 to $100 \%$ $[13,15,16]$.

For this study, we chose to perform only a single measurement using a liver attenuation value $\leq 40 \mathrm{HU}$, since the comparison of hepatic attenuation with splenic attenuation is a more complex method, requiring more time, effort and possibly not contributing to accurate prediction of fat content [17]. The measurement in the liver was performed at just one slice level. This is supported by data showing that fat deposition in the liver is relatively homogeneous and most of 
the variation in the measurement of attenuation in this organ can be captured by measuring it at just one slice [18]. In this study we sought for more homogeneous areas of the liver parenchyma, avoiding vessels, biliary tree or focal lesions [16]. In order to do that, we chose to place the single ROI measurement-with an area of approximately $10 \mathrm{~cm} 2-$ in the right liver lobe, preferentially between the segments VI and VII, because it could proportionate the more suitable area with these characteristics for evaluation (Fig. 1).

Imaging analyses were performed by two readers (Readers 1 and 2, with 5 and 3 years of experience in abdominal imaging, respectively), who were blinded to clinical and laboratory data of all individuals and not involved with patients' selection. Each reader evaluated a portion of the cohort (Reader 1, 121 cases and 61 controls; Reader 2, 83 cases and 51 controls).

\section{Statistical analysis}

The data were described as mean and standard deviation for quantitative variables and frequency tables for qualitative variables. For qualitative variables, absolute frequencies and relative frequencies were used. Confidence intervals were calculated when appropriate. To verify the relationship of variables with "PCR COVID", we applied the Chi square test for qualitative variables and Student's $t$ test for quantitative variables. The variables that by the univariate analysis showed a $p$ value $\leq 20 \%$ were tested together to a logistic regression model. We also conducted post hoc power analyses of our results by using Phi-coefficient. A $p$-value $<0.05$ was considered significant. The data were tabulated using the REDCap platform and analyzed using the SPSS v25 software.

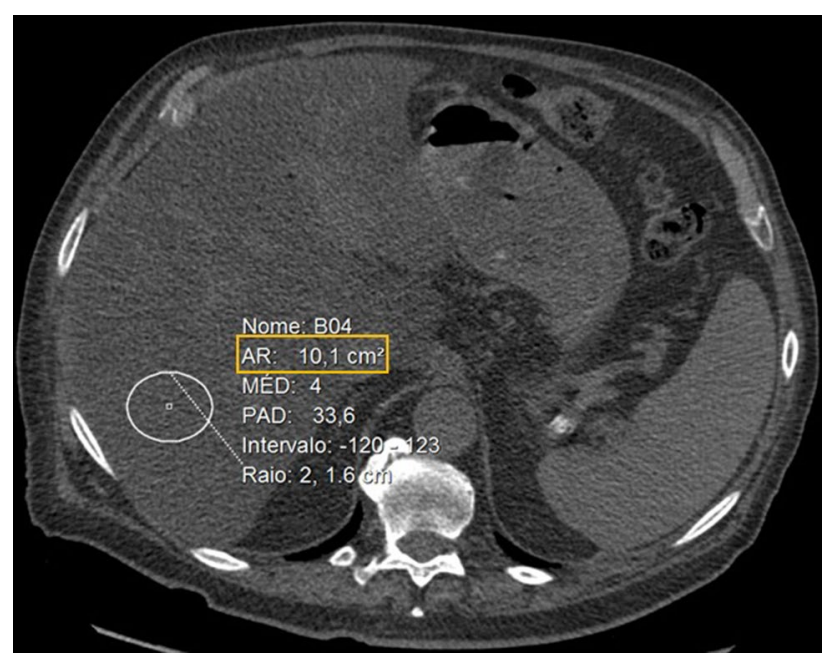

Fig. 1 Demonstration of single ROI positioning and measurementwith an area of approximately $10 \mathrm{~cm}^{2}$-in the right liver lobe, preferentially between the segments VI and VII

\section{Results}

\section{Characteristics of study groups}

A total of 316 individuals participated in the study, including 204 patients with positive RT-PCR for Covid-19 (case group) and 112 with RT-PCR negative in association with chest CT negative pattern (control-group).

The univariate analysis (Table 1) showed that the mean age of the participants was $52.6 \pm 17$ years in the positive group and $43.3 \pm 17$ years in the control group $(p<0.001)$. The sample of patients was composed by 193 women (61\%) and 123 men (39\%). In both groups the number of women was higher, but a higher proportion of men was found in the case group in comparison to the control and a higher proportion of women was found in the control group in comparison to the case group (53.9\% of female vs. $46.6 \%$ of males in cases; $75 \%$ of female vs. $25 \%$ in males in controls; $p<0.001)$.

\section{Association with steatosis}

The frequency of steatosis on CT was higher in the RTPCR positive group than in the control group $(31.9 \%$ vs. $7.1 \%, p<0.001)$. The mean attenuation value in the case group was $46.79 \pm 12.68$ and $53.34 \pm 10.28$ in the control group $(p<0.001)$. The logistic linear regression analysis (Table 2) demonstrated that after adjusting for age and sex there was almost a 4.7 times odds of steatosis in the COVID19 positive group in comparison to controls (OR 4.698; $95 \%$ IC $2.12-10.41, p<0.001)$. For every one year of increase,

Table 1 Comparison of demographic parameters and data on steatosis between the two groups

\begin{tabular}{llll}
\hline Parameter & Positive RT-PCR & $\begin{array}{l}\text { Negative RT-PCR/ } \\
\text { Negative chest CT } \\
(N=112)\end{array}$ & $p$-value \\
& $(N=204)$ & $43.29( \pm 17)$ & $<\mathbf{0 . 0 0 1}$ \\
\hline $\begin{array}{l}\text { Age, years } \\
\text { (median) }\end{array}$ & $52.57( \pm 17)$ & & $<\mathbf{0 . 0 0 1}$ \\
$\begin{array}{l}\text { Sex, } n(\%) \\
\text { Male }\end{array}$ & $95(46.6 \%)$ & $28(25 \%)$ & \\
Female & $109(53.9 \%)$ & $84(75 \%)$ & $<\mathbf{0 . 0 0 1}$ \\
$\begin{array}{l}\text { Steatosis, } n(\%) \\
\text { Presence }\end{array}$ & $65(31.9 \%)$ & $8(7.1 \%)$ & \\
$\quad$ Absence & $139(68.1 \%)$ & $104(92.9 \%)$ & \\
ROI, HU (median) & $46.79 \pm 12.7$ & $53.34 \pm 10.3$ & $<\mathbf{0 . 0 0 1}$ \\
\hline
\end{tabular}

$N$ Number

* $p$ value of less than 0.05 is highlighted in bold

All $p$-values of reports comparisons were analyzed by the Chi square test for qualitative variables and Student's t test for quantitative variables 
Table 2 Logistic linear regression results with odds ratio and 95\% confidence interval values for steatosis and COVID-19, also discriminated according to age and sex

\begin{tabular}{lllr}
\hline Parameter & Odds Ratio & 95\% Confidence interval & $p$ value* \\
\hline Age & 1.026 & $(1.01-1.041)$ & 0.001 \\
Sex (female) & 1.978 & $(1.151-3.40)$ & 0.014 \\
Steatosis & 4.698 & $(2.120-10.410)$ & $<0.001$ \\
\hline
\end{tabular}

The variables that by the univariate analysis showed a $p$-value $\leq 20 \%$ were tested together to a logistic regression model

In variable sex the reference was "female"; in variable steatosis the reference was "negative RT-PCR and negative chest CT",

* $p$ value of less than 0.05 is highlighted in bold

the odds of positive RT-PCR was increased in $2.6 \%$ (OR 1.026 ; $95 \%$ IC $1.01-1.041, p<0.001)$. Women had almost two times the odds for positive RT-PCR (OR 1.978; 95\% IC $1.151-3.40, p<0.014)$. Post hoc power analyses of these results indicated a $99.9 \%$ power of the test, with an effect size of 0.28 ( $p$-value set at 0.05 ).

\section{Discussion}

This is the first study to suggest, to our knowledge, a possible association between hepatic steatosis and COVID-19 infection, with a higher frequency of this liver disorder among COVID-19-positive patients. Such results go in the wake of several previous studies demonstrating the critical relationship between obesity and COVID-19, as hepatic steatosis and obesity are interlinked within a continuum of metabolic abnormalities triggering numerous mechanisms that may be involved in the COVID-19 pathogenesis and increase the vulnerability to infections [3, 5-7].
The global emergence of the obesity epidemic has serious adverse health effects, being the most significant single risk factor for the development of fatty liver, both in children and in adults [19]. Evidence has lately emerged regarding an increased risk of SARS-CoV-2 with worse prognosis in patients with obesity, especially among the young.

It is hypothesized that the increased burden of adipose tissue in obesity may amplify the pro-inflammatory response to viral infection owing to direct, indirect and epigenetic mechanisms, ranging from immune system activity attenuation to chronic inflammation (Fig. 2) [6]. First, there is in obesity a chronic low-grade systemic inflammation (pro-inflammatory state), characterized by increased proinflammatory cytokine secretion from the adipose tissue (especially adipokines, the fat tissue-associated cytokines) [6]. Obese individuals usually also have insulin resistance, reduced beta-cell function, as well chronically higher leptin and lower adiponectin concentrations, an unfavorable profile that may lead to a functional immunologic deficit, as the integrated regulation of metabolism required for the complex cellular interactions, and for effective host defense, are lost [20-22]. Imbalanced activity of the renin-angiotensin-aldosterone system in obese people also seem to have a role in this pathogenesis, as it is also known that SARS-CoV-2 penetrates human cells through binding with angiotensin-converting enzyme 2 (ACE2) [2]; although ACE2 in lung tissue is considered to be a key entry site of the SARS-CoV-2, ACE2 expression in adipose tissue is thought to be even higher than in the lungs, increasing vulnerability of obese people to SARS-CoV-2 [2]. Moreover, B- and T-cell responses are impaired in obese individuals, with numerical and functional alterations of lymphocytes, which may increase susceptibility to viral infection [20]. Under viral infection, in turn, the obesityrelated dysregulated proinflammatory response also leads to reduced macrophage activation (which may explain the poor
Fig. 2 In this scheme, the fatty liver, demonstrated at the hepatocyte level, releases a cascade of systemic inflammatory events triggered by the virus, which may lead to a functional immune deficit. An uncontrolled immune response induces immunopathogenesis, resulting in lung tissue damage and multisystemic functional impairment. Therefore, obesity/ steatosis may serve as a "preactivation state" for immune amplification by SARS-CoV2

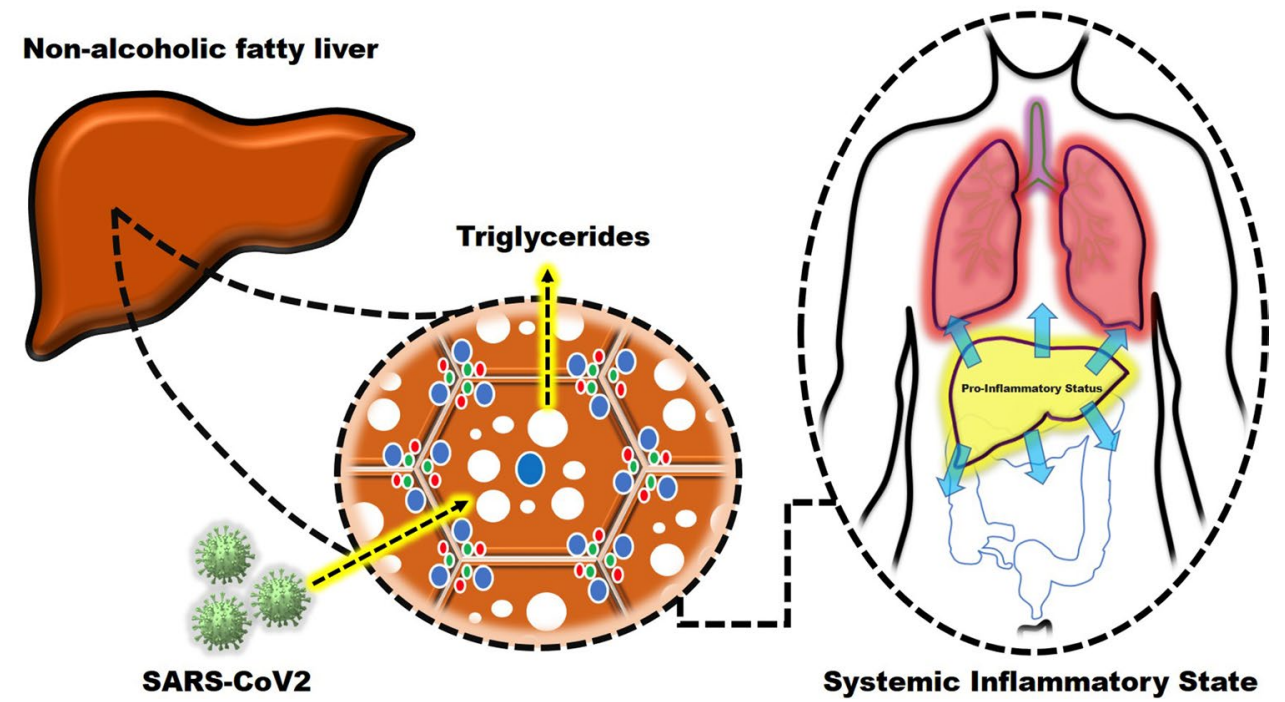


vaccination response in obese patients) [20]. Finally, obesity may increase antiviral resistance [6]. In short, all these factors make the adipose tissue, particularly the visceral adipose tissue, to be pro-immunogenic and metabolically active, capable of driving acute disease through augmented inflammation at an organ level [21].

The capability of COVID-19 driving acute disease in a systemic-wide spectrum may be demonstrated in terms of its abdominal manifestations, for example. Recent studies have been demonstrating that COVID-19 infection can present primarily with abdominal symptoms, such as diarrhea, nausea, vomiting, abdominal pain, and loss of appetite [23, 24]. A study including 1141 confirmed COVID-19 cases found that up to $37 \%$ of patients had abdominal manifestations, and a non-negligible portion ( $16 \%$, or 183 cases) even presented with gastrointestinal symptoms exclusively [23]. An imaging study that assessed 134 patients with available imaging data (224 abdominal imaging exams) found bowel wall abnormalities on $31 \%$ of CT scans, including pneumatosis or portal venous gas, and signs of cholestasis on 54\% of right upper quadrant ultrasounds [24].

CT was the modality of choice employed to assess steatosis in the present study because of its convenience in our specific context (chest CT examinations in patients with flu symptoms in an acute setting). Other advantages of CT include the more recent advent of several techniques capable of promoting dose reduction while maintaining acceptable diagnostic quality (such as automatic exposure control and tube current modulation) or enhancing image quality of reduced-dose $\mathrm{CT}$ images with filtered back projection and iterative reconstruction techniques [25]. However, it should be emphasized the important role that MRI has in the noninvasive diagnosis and staging of liver steatosis and fibrosis, especially when considering that in many cases both conditions may coexist $[26,27]$.

In the present case-control study, we evaluated the association of steatosis on CT with COVID-19 in patients with clinical suspicion for this disease. The discernment of factors predisposing the population to development of COVID19 infection is pertinent for the implementation of public health measures that includes social distancing reinforcement and preventive actions in the future (like changing dietary habit for patients with metabolic syndrome). Indeed, several authors are now recommending assertive diagnostic and therapeutic strategies for obese patients, with systematic screening, careful monitoring and more aggressive treatments $[3,7]$.

In our study, the prevalence of steatosis on CT in the confirmed cases was significantly higher than in the control group, this association being maintained after adjustments for age and sex, which reveals a potential risk of development of infection compared to the group of patients who sought for hospital attendance with respiratory symptoms but were most probably free of infection at that moment. Considering the close relation between steatosis and metabolic syndrome/obesity, our results are in keeping with other studies, including retrospective analyses and cohorts. In a study performed in New York, there was a greater risk for hospital admission in the acute care for patients with a body mass index $(\mathrm{BMI}) \geq 30$ and greater risk for intensive care unit for those with a BMI $\geq 35$ [28]. In an another study, from China, an increased likelihood (fourfold) of severe forms of COVID-19 was demonstrated in patients with metabolic associated fatty liver disease (MAFLD). [29]. As in our case, such study used CT for the diagnosis of steatosis and subsequently diagnosed MAFLD according to other parameters [29].

In our demographic analysis we found a predominance of females in both groups (cases and controls). This corroborates with the profile found in other studies published in Brazil [30] whereas in Wuhan, China, $56 \%$ of the cases were male individuals [31]. It is emphasized that Brazilian population estimative for 2020 shows a slight female dominance [32]. However, when we compared the proportion of the genders in the two groups, we had a higher proportion of males in the case group and a higher proportion of females in the control group, which was statistically significant. After the variables were matched, almost two times odds of men having the disease was seen. Most of the studies that include gender are focused on prognosis (severity and mortality), with one study from China demonstrating that men are more prone to a higher severity of disease [33].

The median age of cases was higher in the confirmed cases in comparison to the control group, which was statistically significant, with an average patient age of 52.6 years, in agreement with a systematic review including 48,926 confirmed COVID-19 cases, in which the average age of patients was 49.2 years [34].

Our study had some limitations. First, we have not assessed other important variables (such as diabetes, hypertension, obesity, weight, height, body mass index, lipid profile and liver function tests), most of which were not available at the patients' charts in our cohort (composed primarily by patients evaluated in an acute/ emergency setting specifically owing to flu symptoms); therefore, we could not establish correlations between such variables and liver steatosis or establish liver steatosis as an independent risk factor for COVID-19 infection. Second, as in many cases the patients had only a single passage by our institution, and even most positive cases were referred to external follow-up (through Telemedicine or with patients' personal doctors, for example), we were unable to have sufficient follow up data on hospitalizations, disease severity or death (some patients may have been admitted in other hospitals at a given moment, for example, which makes incomplete the available data 
on hospitalizations at our institution). Therefore, while previous studies have assessed the relationship between obesity and severity, hospitalization or mortality due to COVID-19, we evaluated only the association between diagnosis of COVID-19 and presence of steatosis, and further studies should be designed to answer if an association exists between steatosis and severity/mortality. Third, as no liver histology was available to compare with CT findings, once CT sensitivity rises to $93 \%$ for detecting steatosis involving greater than $33 \%$ of the liver (moderate cases), probably we can have a bias underestimating the diagnosis of mild steatosis [14]. Fourth, our imaging analysis was performed by two radiologists, which could bring an interobserver bias to our study. However, it is worth mentioning that strict criteria adopted for standardization of measurements and the similar profiles of reader's expertise may have contributed to minimize this possible bias. Finally, despite the difference between both groups in terms of sample size owing to our stricter inclusion criteria for negative individuals (which led to the exclusion of further 84 RT-PCR negative patients) and limitations imposed by the fact that a substantial proportion of RT-PCR negative patients with mild symptoms usually do not undergo a chest CT study as part of their standard management in our institution, post hoc power analyses of our results indicated a high statistical power with this final proportion of included individuals.

Major strengths include the fact that this is the first study to compare steatosis on CT between positive and negative COVID-19 patients; the sample size, which has allowed statistical power to identify the higher frequency of liver steatosis among SARS-CoV2 positive patients; finally, as CT studies were performed on the same scanner (to avoid exposure of patients without flu syndrome), with a variable protocol according to their body habitus, inter-scanner variability that could have affected measurements did not compromise the results, allowing uniformity.

In conclusion, our study shows a significantly higher prevalence of steatosis on CT in confirmed COVID-19 patients as compared to controls after matching to age and sex. These findings, considering the relationship between steatosis, metabolic syndrome and obesity, are in accordance with other recent studies linking obesity and COVID-19 infection. This is of interest for radiologists as liver steatosis can be easily assessable and reported by any radiologist reading a chest CT study in this context, and such information may be integrated to the list of clinical data made available to clinicians, if confirmed to be of clinical relevance. Additional studies are required to confirm if such association remains after accounting for multiple variables, as well as possible relationships with severity and worst clinical outcomes.
Funding This research received no specific grant from any funding agency in the public, commercial, or not-for-profit sectors.

\section{Compliance with ethical standards}

Conflict of interest There are no conflicts of interest to declare.

\section{References}

1. Watanabe M, Risi R, Tuccinardi D, Baquero CJ, Manfrini S, Gnessi L. Obesity and SARS-CoV-2: a population to safeguard. Diabetes Metab Res Rev 2020. https://doi.org/10.1002/dmrr.3325.

2. Lavie CJ, Sanchis-Gomar F, Henry BM, Lippi G. COVID-19 and obesity: links and risks. Expert Rev Endocrinol Metab 2020. https ://doi.org/10.1080/17446651.2020.1767589.

3. Sanchis-Gomar F, Lavie CJ, Mehra MR, Henry BM, Lippi G. Obesity and Outcomes in COVID-19: When an Epidemic and Pandemic Collide. Mayo Clin Proc 2020. https://doi.org/10.1016/j. mayocp.2020.05.006.

4. Murugan AT, Sharma G. Obesity and respiratory diseases. Chron Respir Dis 2008;5:233-42. https://doi.org/10.1177/1479972308 096978.

5. Ryan DH, Ravussin E, Heymsfield S. COVID 19 and the Patient with Obesity - The Editors Speak Out. Obesity 2020;28:847. https ://doi.org/10.1002/oby.22808.

6. Petrakis D, Margină D, Tsarouhas K, Tekos F, Stan M, Nikitovic D, et al. Obesity - a risk factor for increased COVID-19 prevalence, severity and lethality (Review). Mol Med Rep 2020;22:919. https://doi.org/10.3892/mmr.2020.11127.

7. Kassir R. Risk of COVID-19 for patients with obesity. Obes Rev 2020;21:10-1. https://doi.org/10.1111/obr.13034.

8. Dietrich P, Hellerbrand C. Non-alcoholic fatty liver disease, obesity and the metabolic syndrome. Best Pract Res Clin Gastroenterol 2014;28:637-53. https://doi.org/10.1016/j.bpg.2014.07.008.

9. Hamer OW, Aguirre DA, Casola G, Sirlin CB. Imaging features of perivascular fatty infiltration of the liver: Initial observations. Radiology 2005;237:159-69. https://doi.org/10.1148/radiol.23710 41580 .

10. Yang W, Sirajuddin A, Zhang X, Liu G, Teng Z, Zhao S, et al. The role of imaging in 2019 novel coronavirus pneumonia (COVID19). Eur Radiol 2020. https://doi.org/10.1007/s00330-020-06827 -4 .

11. Simpson S, Kay FU, Abbara S, et al. Radiological Society of North America Expert Consensus Statement on Reporting Chest CT Findings Related to COVID-19. Endorsed by the Society of Thoracic Radiology, the American College of Radiology, and RSNA. J Thorac Imaging. 2020;https://doi.org/10.1097/rti.00000 00000000524. https://doi.org/10.1097/rti.0000000000000524

12. Lawrence DA, Oliva IB, Israel GM. Detection of hepatic steatosis on contrast-enhanced CT images: Diagnostic accuracy of identification of areas of presumed focal fatty sparing. Am J Roentgenol 2012;199:44-7. https://doi.org/10.2214/AJR.11.7838.

13. Monjardim RDF, Costa DMC, Romano RFT, Salvadori PS, dos Santos J de VC, Von Atzingen AC, et al. Diagnosis of hepatic steatosis by contrast-enhanced abdominal computed tomography. Radiol Bras 2013;46:134-8. https://doi.org/10.1590/S0100-39842 013000300005.

14. Wells MM, Li Z, Addeman B, Mckenzie CA, Mujoomdar A, Beaton M, et al. Computed Tomography Measurement of Hepatic Steatosis: Prevalence of Hepatic Steatosis in a Canadian Population. Can J Gastroenterol Hepatol 2016;2016:4930987. https://doi. org/10.1155/2016/4930987. 
15. Ma X, Holalkere NS, Avinash KR, Mino-Kenudson M, Hahn PF, Sahani D V. Imaging-based quantification of hepatic fat: Methods and clinical applications. Radiographics 2009;29:1253-77. https ://doi.org/10.1148/rg.295085186.

16. Boyce CJ, Pickhardt PJ, Kim DH, Taylor AJ, Winter TC, Bruce $\mathrm{RJ}$, et al. Hepatic steatosis (fatty liver disease) in asymptomatic adults identified by unenhanced low-dose CT. Am J Roentgenol 2010;194:623-8. https://doi.org/10.2214/AJR.09.2590.

17. Kodama Y, Ng CS, Wu TT, Ayers GD, Curley SA, Abdalla EK, et al. Comparison of CT methods for determining the fat content of the liver. Am J Roentgenol 2007;188:1307-12. https://doi. org/10.2214/AJR.06.0992.

18. Speliotes EK, Massaro JM, Hoffmann U, Foster MC, Sahani D V., Hirschhorn JN, et al. Liver fat is reproducibly measured using computed tomography in the Framingham Heart Study. J Gastroenterol Hepatol 2008. https://doi.org/10.111 1/j.1440-1746.2008.05420.x.

19. Festi D, Colecchia a, Sacco T, Bondi M, Roda E, Marchesini G. Hepatic steatosis in obese patients : clinical aspects and prognostic significance. Obes Rev 2004;5:27-42. https://doi.org/10.1111/ j.1467-789x.2004.00126.x.

20. Kim J, Nam JH. Insight into the relationship between obesityinduced low-level chronic inflammation and COVID-19 infection. Int J Obes (Lond) 2020;44(7):1541-1542. https://doi.org/10.1038/ s41366-020-0602-y

21. Ryan PM, Caplice NM. Is Adipose Tissue a Reservoir for Viral Spread, Immune Activation and Cytokine Amplification in COVID-19. Obesity (Silver Spring) 2020. https://doi.org/10.1002/ oby. 22843.

22. Sattar N, McInnes IB, McMurray JJV. Obesity a Risk Factor for Severe COVID-19 Infection: Multiple Potential Mechanisms. Circulation 2020;https://doi.org/10.1161/circulationaha.120.047659. https://doi.org/10.1161/circulationaha.120.047659

23. Luo S, Zhang X, Xu H. Don't Overlook Digestive Symptoms in Patients With 2019 Novel Coronavirus Disease (COVID-19). Clin Gastroenterol Hepatol 2020;18(7):1636-1637. https://doi. org/10.1016/j.cgh.2020.03.043

24. Bhayana R, Som A, Li MD, et al. Abdominal Imaging Findings in COVID-19: Preliminary Observations. Radiology 2020;201908. https://doi.org/10.1148/radiol.2020201908

25. Elmokadem AH, Ibrahim EA, Gouda WA, et al. Whole-Body Computed Tomography Using Low-Dose Biphasic Injection Protocol With Adaptive Statistical Iterative Reconstruction V: Assessment of Dose Reduction and Image Quality in Trauma Patients. J Comput Assist Tomogr 2019;43(6):870-876. https:// doi.org/10.1097/rct.0000000000000907
26. Besheer T, Razek AAKA, El Bendary M, et al. Does steatosis affect the performance of diffusion-weighted MRI values for fibrosis evaluation in patients with chronic hepatitis $C$ genotype 4 ? Turk J Gastroenterol 2017;28(4):283-288. https://doi.org/10.5152/ tjg.2017.16640

27. Besheer T, Elalfy H, Abd El-Maksoud M, et al. Diffusionweighted magnetic resonance imaging and micro-RNA in the diagnosis of hepatic fibrosis in chronic hepatitis $\mathrm{C}$ virus. World J Gastroenterol 2019;25(11):1366-1377. https://doi.org/10.3748/ wjg.v25.i11.1366

28. Lighter J, Phillips M, Hochman S, Sterling S, Johnson D, Francois $\mathrm{F}$, et al. Obesity in patients younger than 60 years is a risk factor for Covid-19 hospital admission. Clin Infect Dis 2020:2019-20. https://doi.org/10.1093/cid/ciaa415.

29. Gao F, Zheng KI, Wang X-B, Yan H-D, Sun Q-F, Pan K-H, et al. Metabolic associated fatty liver disease increases COVID-19 disease severity in non-diabetic patients. J Gastroenterol Hepatol 2020. https://doi.org/10.1111/jgh.15112.

30. Almeida JS, Cardoso JA, Cordeiro EC, Araújo TME De, Sardinha AHDL. [Epidemiological characterization of COVID-19 cases in Maranhão: a brief analysis]. Scielo Preprints 2020. https://doi. org/10.1590/SciELOPreprints.314

31. Li Q, Guan X, Wu P, Wang X, Zhou L, Tong Y, et al. Early transmission dynamics in Wuhan, China, of novel coronavirus-infected pneumonia. N Engl J Med 2020. https://doi.org/10.1056/NEJMo a2001316.

32. Brazilian Ministry of Health. Projeção da População do Brasil por sexo e idade simples: 2000-2060. 2020. Available from: http:// tabnet.datasus.gov.br/cgi/tabcgi.exe?ibge/cnv/projpopbr.def. Accessed on June 102020.

33. Jin JM, Bai P, He W, Wu F, Liu XF, Han DM, et al. Gender Differences in Patients With COVID-19: Focus on Severity and Mortality. Front Public Heal 2020;8:1-6. https://doi.org/10.3389/ fpubh.2020.00152.

34. Tan SC. Clinical and epidemiological characteristics of Coronavirus Disease 2019 (COVID-19) patients. MedRxiv 2020:2020.04.02.20050989. https://doi. org/10.1101/2020.04.02.20050989.

Publisher's Note Springer Nature remains neutral with regard to jurisdictional claims in published maps and institutional affiliations. 